- hospital and area health service employees performing their existing roles but having additional or altered reporting requirements for the duration of the Games;

- Department of Health staff employed on a temporary basis to provide Games services, or permanent staff redeployed to Games roles for the duration.

A significant component of the NSW Health Olympic Workforce has been drawn from local government, a commitment that is unprecedented in its scale and which may serve as a model for future cooperative efforts.

\section{COORDINATION MECHANISMS}

In order to monitor health service use and to facilitate strategic responses to health issues as they arise, the NSW Health Olympic Coordination Centre (HOCC) will be established at 73 Miller Street, North Sydney. The existing Olympic Planning Unit will provide the core staff for the Centre and service its intelligence gathering and response requirements.

HOCC will have a 24-hour contact number, and will be staffed by duty officers from 2 September to 1 November 2000, to facilitate liaison between the Department, SOCOG, OCA, hospitals and area health services on operational issues as they arise. Figure 1 illustrates the linkages between the HOCC and other key health coordination centres active at the time of the Games. Important functions of HOCC will include:

- activation of coordinated public health responses. This will be informed by the Olympic Surveillance System (see the article by Thackway on page 142) and based on recommendations of a Surveillance Review Team which will convene daily, preparatory to a daily HOCC meeting. Figure 2 illustrates the HOCC reporting and communications linkages with various components of the health system.

- coordination of media responses.

\section{CONCLUSION}

The Olympic Athlete's Village will soon open, and this will activate NSW Health operational plans for the Games. There is a buzz of excitement that after four years of active planning, the Games are finally here. Hopefully all those in NSW Health who have worked so hard to prepare for the event will be able to take the advice of an Atlanta colleague: 'Remember to take some time and enjoy the Olympic experience! I know how fast it has arrived, AND how quickly it will be over.'

\title{
COUNTER DISASTER PLANNING FOR THE SYDNEY 2000 OLYMPIC AND PARALYMPIC GAMES
}

\section{Michael Flynn \\ Director, Counter Disaster and Olympic Planning Branch NSW Department of Health}

Sydney's submission for its 1992 bid to host the Summer Games of the XXVII Olympiad included the following statement: 'Sydney has no history of natural disasters, so disaster planning for the Olympics has been based on the New South Wales Multiple Casualty, Emergency and Disaster Medical Response Plan (MEDPLAN). ${ }^{1}$

Much progress has been made in the field of disaster medicine policy development since this original submission. The concept of 'disaster' has been expanded to encompass such non-traditional events as loss of utility supply and technological failure. The importance of the media has been given increased recongition.

\section{DISASTER MEDICINE AND ITS ROLE IN PUBLIC HEALTH}

Within NSW Health the counter disaster plan, MEDPLAN, was in the process of being replaced by a substantially revised policy (retitled HEALTHPLAN) at the time of the
Thredbo landslide (31 July 1997)., ${ }^{2,3}$ This tragedy, which resulted in the loss of 18 lives, involved a significant deployment of emergency workers, including health workers, in a challenging environment over several weeks. Lessons learned from this disaster affirmed the importance of the roles of public health, mental health and ambulance services in the emergency response, as well as those of other participating and supporting organisations within the disaster plan. Concurrently, the role and resources of the Counter Disaster Unit in the Public Health Division of the NSW Department of Health were expanded.

\section{DISASTER PLANNING FOR THE SYDNEY 2000 OLYMPIC AND PARALYMPIC GAMES}

The Olympic Games is arguably the most significant mass gathering in the world. Although relatively rare, mass gatherings have been associated with significant morbidity and death. Examples include crowd crushes at Hillsborough Stadium in the United Kingdom, the collapse of a pedestrian bridge at the 1997 Maccabiah Games in Israel, and terrorist activities at the Munich Games in $1972 . .^{4,5}$ The blue glass memorial, inscribed in English and Hebrew, on the 'Munich XX Olympiad' 
solar light tower located on the Olympic Boulevard at Homebush Bay, serves as a reminder of the potential for non-accidental disasters to be superimposed on the complexities inherent in planning for such an event.

Planning for such a contingency requires the commitment of significant resources to the development of new treatment protocols, to training and exercises, and to the acquisition of personal protective equipment (PPE) and specialised pharmaceuticals hitherto used primarily by the military. It has also involved structural modifications of selected public hospital emergency departments to ensure preservation of their ability to deliver their core functions.

Disaster planning for the Games has also required a degree of public engagement through the media to place into perspective some of the more extreme scenarios canvassed by various groups. ${ }^{6,7}$ Reference to previous Australian experience with putative or potential, natural or 'man-made' threats to public health has facilitated this engagement process. ${ }^{8}$

Around 100 NSW Health personnel and 30 from other agencies have undertaken advanced disaster medicine training in the 18-month period January 1999-June 2000. A further 220 personnel have undertaken multi-agency and specially tailored Health Aspects of Chemical, Biological and Radiological (CBR) response training. Material from both groups of courses has been made available to participants to enable them to provide general awareness training to other NSW Health personnel. The enhancement of the State's disaster medicine capability to meet hitherto unmet needs for the optimal management of a wide range of industrial and agricultural hazardous materials (HAZMAT) will be one of the Olympic legacies.

From an urban mass gathering perspective, the Games have been likened to ' 15 consecutive New Year's Eve-New Year's Day celebrations'. During September, pre-formed Disaster Medical Response Teams will be available to be deployed to any site in the Sydney metropolitan area to support the designated 'combat' agencies in the field. Counter Disaster Unit staff have participated in a range of exercises to test and refine their response protocols. The Sun-Herald City to Surf race on 16 July 2000 in support of around 50,000 runners was utilised as one of the final exercises prior to the Games.

\section{LIAISON}

An extensive consultative process with interested stakeholders has been crucial to the NSW Health planning process for the Games. This has included consultations with:

- international, commonwealth (including the Australian Defence Force), state and territory health agencies

- the Olympic Coordination Authority

\section{- SOCOG-SPOC}

- the State Emergency Management Committee

- relevant 'combat' agencies including the Ambulance Service of NSW, NSW Fire Brigades and the NSW Police Service

- professional health networks (such as the communicable disease networks of Australia and New Zealand, and the public health laboratories network)

- NSW forensic services

- NSW area health services (via Health Service Functional Area Coordinators) and their Olympic Planning Committees

- local government authorities

- the City of Sydney Olympic Coordination Committee

- the Protocol Section of the Premier's Department (health support for visiting dignitaries).

\section{COORDINATION, COMMAND, CONTROL AND COMMUNICATION (C4)}

The ability to communicate information quickly and accurately lies close to the core business of any major organisation, especially health. Aligned to this is the need to have highly visible lines of command and control. Within NSW Health, the Counter Disaster Unit has developed a framework to ensure close links with external operations centres (the Olympic Security Command Centre, Olympic Precinct and Regional Operations Centre, and the State Emergency Operations Centre) and rapid dissemination of information to area health services, units and supporting networks.

New Year's Eve-New Year's Day 1999-2000 (Y2K) Transition Planning served to facilitate an unprecedented degree of cooperation and communication between a wide range of government and non-government agencies. It also served as a medium for accelerating the commissioning of a new Health Services Disaster Control Centre (HSDCC) at Rozelle. For the first time, Web-based electronic information transfer was employed and this technology has been enhanced (Health Incident Reporting Information System, or HIRIS) and will be utilised during the Games. The HSDCC will be linked to all other Operations Centres including the NSW Health Olympic Coordinating Centre (HOCC) in North Sydney. NSW Health Public Affairs has worked closely with media representatives from other agencies to ensure that information will be provided in a timely and accurate manner.

NSW Health's involvement in the response to the April 1999 Sydney hailstorm, and the activation of the Commonwealth Plan for the Reception of Refugees for the Kosovar and East Timorese refugee groups also provided invaluable operational experience. 


\section{SUMMARY AND CONCLUSION}

Five days after Sydney's successful Olympic and Paralympic bid application was announced in Monte Carlo, the first meeting of the Australian Medical Disaster Coordination Group (AMDCG), ${ }^{9}$ a sub-group of the Australian Health Ministers Advisory Council (AHMAC) was held at Alice Springs. The seventh AMDCG meeting was held recently in Melbourne (2-4 April 2000). The successful collaborative outcomes of this seven-year period reported to AHMAC include authoritative and up to date publications in the fields of disaster medicine, ${ }^{10}$ mass gatherings, ${ }^{11}$ and chemical, biological and radiological hazards. ${ }^{12}$

NSW Health has devoted substantial resources to the accepted emergency management principles of planning, preparation and prevention outlined in these and other documents, in progressively developing its strategic plan for service delivery during the Games.

Counter disaster planning, expressed through NSW HEALTHPLAN and supporting Olympic Games Standing Operating Procedures, has been framed to ensure that an appropriate and effective response can be mounted in the event of any untoward happening.

\section{REFERENCES}

1 NSW Government Olympic Bid Document. Sydney: NSW State Government, 1992; Para 7.2.4.

2 NSW Department of Health. New South Wales Multiple Casualty, Emergency and Disaster Medical Response Plan (MEDPLAN). Sydney: NSW Department of Health, July, 1990.
3 NSW Department of Health. NSW Health Services HEALTHPLAN-Functional Area Supporting Plan to the New South Wales State Disaster Plan (NSW DISPLAN). Sydney: NSW Department of Health, December 1996. State Health Publication Number PH960098.

4 Daley, P. Terror alert on Anthrax. Sunday Age, Melbourne, 4 June 2000.

5 Clancy T. Rainbow 6. Melbourne: Penguin Books, 1998.

6 International Union of Microbiological Societies. Proceedings of the Bioterrorism Symposium, 14 August 1999, Darling Harbour Convention Centre NSW. Audio cassettes. Padstow, Sydney: Quickopy Audio Recording Services, 1999.

7 Evangeli A. Disaster Planning-Bioterrorism and the Olympics. Medicine Today 1 July 2000: 148-153.

8 Flynn MJ. Editorial: Sydney and the Games: what are the risks? Microbiology Australia September 1999: (20)4.

9 Department of Health Housing, Local Government and Community Services. Report of the Australian Medical Disaster Coordination Group Meeting, Alice Springs 2830 September 1993. Canberra: Commonwealth of Australia, 1993.

10 Emergency Management Australia, Department of Defence. Australian Emergency Manuals Series Part III, Volume 1 Manual 2, Disaster Medicine. Canberra: Commonwealth of Australia, 1999.

11 Emergency Management Australia, Department of Defence. Australian Emergency Manuals Series Part III, Volume 2 Manual 2, Safe and Healthy Mass Gatherings. Canberra: Commonwealth of Australia, 1999.

12 Emergency Management Australia, Department of Defence. Australian Emergency Manuals Series Part III, Volume 2 Manual 3, Health Aspects of Chemical, Biological and Radiological Hazards (Provisional Edition). Canberra: Commonwealth of Australia, 2000.

\section{HEALTH SURVEILLANCE DURING THE SYDNEY 2000 OLYMPIC AND PARALYMPIC GAMES}

Sarah Thackway,

on behalf of the Olympic Surveillance Working Party

Olympic Planning Unit

NSW Department of Health

This article describes the Olympic Surveillance System developed by NSW Health to monitor the health of the population of Sydney during the Sydney 2000 Olympic and Paralympic Games. The surveillance system will be the most comprehensive ever undertaken in NSW and will provide more coverage than the systems used during previous Olympic Games.

\section{HEALTH SURVEILLANCE DURING MASS GATHERINGS}

Mass gatherings, such as sporting events and outdoor celebrations, require the provision of medical services for the large numbers of people who attend. ${ }^{1-3}$ In addition, the crowding of people into relatively closed environments may promote the transmission of infectious agents spread via the respiratory route (for example, measles and influenza). ${ }^{4-5}$

The provision of medical and public health services is enhanced by epidemiological surveillance, an important tool implemented to monitor unusual patterns of illness. Surveillance systems act as an early warning to detect communicable disease outbreaks that can occur during these gatherings, as well as unusual patterns of noninfectious conditions such as injuries that may require public health intervention. In previous Olympic Games, a variety of surveillance systems have been implemented to monitor for outbreaks of disease. ${ }^{6-8}$ 